\title{
A REFINEMENT OF THE GENERALIZED CHORDAL DISTANCE
}

\author{
AMOL SASANE
}

\begin{abstract}
For single input single output systems, we give a refinement $d_{c, r}$ of the generalized chordal metric $d_{c}$ introduced in 13 . Our metric $d_{c, r}$ is given in terms of coprime factorizations, but it coincides with the extension of Vinnicombe's $\nu$-metric given in Ball and Sasane [1] if the coprime factorizations happen to be normalized. The advantage of the metric $d_{c, r}$ introduced in this article is its easy computability (since it relies only on coprime factorizations, and does not require normalized coprime factorizations). We also give concrete formulations of our abstract metric for standard classes of stable transfer functions.
\end{abstract}

\section{INTRODUCTION}

The Vinnicombe $\nu$-metric introduced in [16] and its abstract version given in [1] both rely on finding normalized coprime factorizations. This is a troublesome aspect of the theory, since

(1) Although merely coprime factorizations may exist, a normalized coprime factorization may fail to exist: for example in the article [14] by Sergei Treil, it was shown that the set of plants in the field of fractions of the disk algebra possessing normalized coprime factorizations is strictly contained in the set of plants possessing coprime factorizations.

(2) Even if they exist, normalized coprime factorizations might be impossible to find using a constructive procedure: for example, in the paper [8] by Jonathan Partington and Gregory Sankaran, it is shown that in the case of delay systems, in general the relevant spectral factorizations for finding normalized coprime factorizations cannot be determined by solving any finite system of polynomial equations over the field $\mathbb{R}\left(s, e^{s}\right)$.

The goal in this paper is to show that this problematic feature of the $\nu$-metric can be eliminated, at least in the case of single input single output systems, by redefining the $\nu$-metric, which relies only on coprime factorizations, rather than normalized coprime factorizations. The starting point is the generalized chordal distance $d_{c}$ introduced in [13] given in terms of coprime factorizations, and then considering a refinement $d_{c, r}$ of this chordal distance akin to the $\nu$-metric $d_{\nu}$. It turns out that the metric $d_{c, r}$ coincides with the $\nu$-metric if one has normalized coprime factorizations at hand. But since our metric is in fact only defined using coprime factorizations, the burden of working with normalized coprime factorizations is completely eliminated. Our main results are then that $d_{c, r}$ defines a metric on the set of all elements admitting a coprime factorization, and stabilizability is a robust property of the plant. The precise statements of the results are given in Theorems 4.3 and 4.5 , after the notation has been introduced in the next section.

1991 Mathematics Subject Classification. Primary 93B36; Secondary 93D15, 46J15.

Key words and phrases. $\nu$-metric, robust control, Banach algebras. 


\section{BACKGROUnd, KNOWN RESUlts AND RELATED LiTERATURE}

Let us recall that in the "factorization approach" to linear control theory, one starts with an integral domain $R$, which is thought to constitute the set of all transfer functions of stable linear systems. Transfer functions of linear systems which are not necessarily stable are then taken to be elements of the field of fractions $\mathbb{F}(R)$ over $R$. Based on algebraic factorizations of the plant transfer function, control theoretic problems can then be posed and solved. We refer the uninitiated reader to the monograph by [15]. This factorization approach to linear control theory has been resurrected and extended in the articles [9], [10], [7] and the references therein. In particular, in [9], [10], a theory of solving the stabilization problem (recalled below) is developed, which does not rely on the existence of coprime factorizations, but instead proceeds under weaker notions of coprimeness. This theme is also present in [7, with further emphasis on obtaining stabilizing controllers that are "real symmetric" (and hence physically implementable) given real symmetric data. Our concern in this article is not with the stabilization problem, but rather the robust stabilization problem. Before recalling the robust stabilization problem, let us first remind ourselves of the stabilization problem.

The stabilization problem is the following: Given $\mathbf{p} \in \mathbb{F}(R)$ (and unstable plant), find $\mathbf{c} \in \mathbb{F}(R)$ (a stabilizing controller) such that the "interconnection" of $\mathbf{p}$ and $\mathbf{c}$ is stable, that is, the closed loop transfer function is stable:

$$
\left[\begin{array}{cc}
\frac{-\mathbf{p c}}{1-\mathbf{p c}} & \frac{\mathbf{p}}{1-\mathbf{p c}} \\
\frac{-\mathbf{c}}{1-\mathbf{p c}} & \frac{1}{1-\mathbf{p c}}
\end{array}\right] \in R^{2 \times 2} .
$$

It is well known that this problem can be solved if $\mathbf{p}$ has a "coprime factorization" [15.

In reality, the plant transfer function is computed from the differential equation model of the situation at hand, which in turn is obtained from a modelling procedure involving idealizations, simplifying assumptions, approximations, and measurement of parameters. This means that the plant transfer function is not known precisely, but serves only as an approximation of reality. Hence engineers imagine that all we know is a "nominal" plant transfer function $\mathbf{p}_{0}$, and the reality might well be a perturbation $\mathbf{p}$ of this nominal transfer function $\mathbf{p}_{0}$. So one wishes the stabilizing controller $\mathbf{c}$ found for the nominal $\mathbf{p}_{0}$ to stabilize not only $\mathbf{p}_{0}$, but also all plants $\mathbf{p}$ close enough to $\mathbf{p}_{0}$, and we would also like to be able to compute the radius of this neighbourhood (so that the engineers are aware of the parametric uncertainity tolerated). The question of what should be an appropriate notion of distance one should use

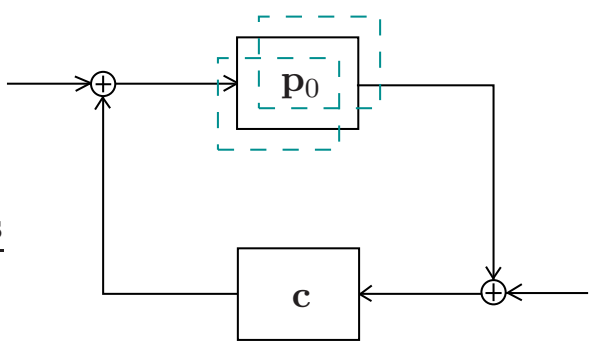

FiguRE 1. Robust stabilization of an uncertain plant.

to measure closeness of unstable plants thus arises naturally. Based on the goals described in 
the above paragraph, it is natural to demand a metric $d$ on the set of stabilizable plants such that

(1) $d$ is easily computable, and

(2) $d$ has the following property with respect to the Stabilization Problem: Stabilizability becomes a robust property of stabilizable plants in this metric. In other words, if $\mathbf{p}_{0}$ is stabilized by a controller $\mathbf{c}$, then there exists an $r>0$ such that every $\mathbf{p}$ in the ball $B\left(\mathbf{p}_{0}, r\right):=\left\{\mathbf{p}: d\left(p, p_{0}\right)<r\right\}$ is stabilized by $\mathbf{p}$, and this $r$ should be easily computable too.

Such a metric was introduced by Vinnicombe in [16] when $R=R H^{\infty}$ (rational functions without poles in the right half plane or the imaginary axis), and was called the $\nu$-metric in [16]. The definition of the $\nu$-metric was given in terms of normalized coprime factorizations. An abstract extension of this metric was given in [1] in order to cover the case when $R \neq R H^{\infty}$, but rather $R$ is the class of stable transfer functions of infinite dimensional systems, for example, when $R$ is the Callier-Desoer algebra $\left(\mathcal{A}^{+}\right.$defined on page 12 in the present article). The starting point in [1] was abstract, where it was assumed that $R$ satisfies some mild assumptions, and then an abstract $\nu$-metric was defined on those elements of $\mathbb{F}(R)$ that possess a normalized coprime factorization. Specific examples of $R$ satisfying the abstract assumptions and the resulting particular examples of the $\nu$-metric were given in [1. Notably, that the case when $R=\mathcal{A}^{+}$is covered by the abstract set up was given in [1, but not the case when $R=H^{\infty}$ (bounded and holomorphic functions in the unit disc with center 0 in $\mathbb{C}$ ). Subsequently, in the auxiliary papers [2], 12] and [5], the abstract $\nu$-metric introduced in [1] was applied in the particular case when $R=H^{\infty}$. [5] was essentially a new reformulation of [12] (where the case $R=H^{\infty}$ was covered first), while [2] covered only the case of $R=Q A$ (quasi analytic functions). In all of these, just like in [1, the $\nu$-metric relied on the existence of normalized coprime factorizations. As pointed out in the introduction, existence of a coprime factorization of a plant does not automatically imply the existence of a normalized one, and even if a normalized one is known to exist, there may not be a computational procedure to find it. On the other hand, the problem of finding coprime factorizations is much more tractable than finding normalized coprime ones, and so it is desirable to find an extension of Vinnicombe's metric which is given on the set of all plants that possess a coprime factorization. It is this issue that is addressed in the present paper. Hence the results in the present work are not a consequence of these previous works. See the schematic diagram on page 4 .

Finally, we remark that in the previous work [13], we showed robustness of the property of strong stabilizability of the "generalized chordal distance" in a somewhat different setting. The point of the present work is that by considering a refinement of the generalized chordal distance from [13, we can show that the robustness of the property of stabilizability. We remark that in general rings $R$ (for example in $R=\mathcal{A}^{+}$), the set of stabilizable plants is strictly bigger than the set of strongly stabilizable plants.

\section{Notation}

For the convenience of the reader, we have included a table here which shows the page numbers of the places where the corresponding notation is first defined. 


\section{Present article: Refinement of the abstract chordal distance} (distance defined using coprime factorizations)

11. Abstract $\nu$-metric for single input single output systems (distance defined using normalized coprime factorizations)

[1] $R=R H^{\infty}$ (Vinnicombe $\nu$-metric)

[1] $R=\mathcal{A}^{+}$

[2] $R=Q A$

$[12] \equiv[5] R=H^{\infty}$

\begin{tabular}{|c|c|}
\hline Notation & Page number \\
\hline$R$ & (A1) on page [5] \\
\hline$S, I$ & (A2) on page 5 \\
\hline.$^{*}$ & involution; (A2) on page 5 and page 14 \\
\hline$\iota, \circ, G$ & (A3) on page [5] \\
\hline $\mathbb{F}(R)$ & field of fractions over $R$; page 5 \\
\hline $\mathbb{S}$ & set of plants having a coprime factorization; page 5 \\
\hline $\mathbb{S}_{n}$ & set of plants having a normalized coprime factorization; page 6 \\
\hline$\widehat{\cdot}$ & Gelfand/Laplace transform, page [5 and page [12 \\
\hline$M(S)$ & maximal ideal space of $S$; page 5 \\
\hline$\|\cdot\|_{S}=\|\cdot\|_{S, \infty}$ & norm in the $C^{*}$ algebra $S$; page 5 \\
\hline$|\cdot|$ & induced operator norm from $\mathbb{C}^{p}$ to $\mathbb{C}^{m} ;$ page 6 \\
\hline$\kappa_{\mathbf{p}_{1}, \mathbf{p}_{2}}$ & chordal distance function between $\mathbf{p}_{1}, \mathbf{p}_{2} ;$ page $[5$ \\
\hline$d_{c, r}$ & refinement of the chordal distance; page 6 \\
\hline$\mu_{\mathbf{p}, \mathbf{c}}$ & stability margin of $(\mathbf{p}, \mathbf{c}) ;$ page 6 \\
\hline $\mathbb{D}, \mathbb{T}, \overline{\mathbb{D}}, \mathbb{C}_{>0}$ & certain subsets of $\mathbb{C}$; page 12 \\
\hline$H^{\infty}$ & the Hardy algebra; page 3 \\
\hline$R H^{\infty}$ & set of rational elements of $H^{\infty}$; page $[12$ \\
\hline$W^{+}(\mathbb{D})$ & the Wiener algebra; page 12 \\
\hline$A(\mathbb{D})$ & the disc algebra; page 12 \\
\hline$L^{\widehat{1}[0, \infty)}+\mathbb{C}$ & the Wiener-Laplace algebra; page 12 \\
\hline$C(\mathbb{T})$ & set of continuous functions on $\mathbb{T}$; page $[12$ \\
\hline$w$ & page 12 and page 14 \\
\hline $\mathcal{A}^{+}$ & page $\lcm{12}$ \\
\hline$C_{0}$ & set of continuous functions on $\mathbb{R}$ vanishing at $\pm \infty$; page 12 \\
\hline$A P$ & set of almost periodic functions; page $[12$ \\
\hline$w_{a v}$ & average winding number; page 13 \\
\hline $\mathbb{A}_{r}, \mathbb{A}_{0}$ & annulus; page 13 \\
\hline$\pi_{r}^{R}$ & restriction; page 13 \\
\hline$\pi_{r}$ & restriction; page 14 \\
\hline$C_{b}\left(\mathbb{A}_{r}\right), \prod_{r \in(0,1)} C_{b}\left(\mathbb{A}_{r}\right)$ & page 13 \\
\hline $\mathcal{A}, N$ & page 13 \\
\hline$\beta \mathbb{A}_{0}, C\left(\beta \mathbb{A}_{0} \backslash \mathbb{A}_{0}\right)$ & page 14 \\
\hline
\end{tabular}




\section{SETUP AND PRELIMINARIES}

Our setup is the following:

(A1) $R$ is commutative integral domain with identity.

(A2) $S$ is a commutative $C^{*}$-algebra such that $R \subset S$, that is there is an injective ring homomorphism $I: R \rightarrow S$.

(A3) Let inv $S$ to denote the invertible elements of $S$. There exists a map $\iota: \operatorname{inv} S \rightarrow G$, where $(G,+)$ is an Abelian group with identity denoted by $\circ$, and $\iota$ satisfies

(I1) $\iota(a b)=\iota(a)+\iota(b)(a, b \in \operatorname{inv} S)$.

(I2) $\iota\left(a^{*}\right)=-\iota(a)(a \in \operatorname{inv} S)$.

(I3) $\iota$ is locally constant, that is, $\iota$ is continuous when $G$ has the discrete topology.

(I4) $\mathbf{x} \in R \cap(\operatorname{inv} S$ ) is invertible as an element of $R$ if and only if $\iota(\mathbf{x})=0$.

(I5) If $\mathbf{x} \in S$ and $\mathbf{x}>0$, then $\iota(\mathbf{x})=0$.

(A3) allows identification of elements of $R$ with elements of $S$. So in the sequel, if $\mathbf{x}$ is an element of $R$, we will simply write $\mathbf{x}$ (an element of $S$ !) instead of $I(\mathbf{x})$. A consequence of (I3) is the following homotopic invariance of the index [1, Proposition 2.1], which we will use in the sequel. (I4) is to be thought of as an abstract Nyquist criterion. We also remark that there are salient differences in the setup in 11 versus the above. In particular, we take $S$ to be a $C^{*}$-algebra, whereas in [1, $S$ was a Banach algebra, and also the condition (I5), which is critically used in our proofs, was missing in the framework of [1].

Proposition 4.1. If $H:[0,1] \rightarrow \operatorname{inv} S$ is continuous, then $\iota(H(0))=\iota(H(1))$.

Proof. This follows from (I3); see [1, Proposition 2.1].

We also introduce the following standard notation and terminology:

$\mathbb{F}(R)$ denotes the field of fractions of $R$. Given $\mathbf{p} \in \mathbb{F}(R)$, a factorization

$$
\mathbf{p}=\frac{\mathbf{n}}{\mathbf{d}}
$$

where $\mathbf{n} \in R, \mathbf{d} \in R \backslash\{0\}$, is called a coprime factorization of $\mathbf{p}$ if there exist $\mathbf{x}, \mathbf{y} \in R$ such that

$$
\mathbf{n x}+\mathbf{d y}=1 .
$$

We denote by $\mathbb{S}$ the set of all elements in $\mathbb{F}(R)$ that possess a coprime factorization.

The maximal ideal space of $S$ is denoted by $M(S)$. If $\mathrm{x} \in S$, then we denote by $\widehat{\mathrm{x}}$ the Gelfand transform of x. Also, by the Gelfand-Naimark Theorem [11, Theorem 11.18],

$$
\|\mathbf{x}\|_{S}=\|\widehat{\mathbf{x}}\|_{S, \infty}=\max _{\varphi \in M(S)}|\widehat{\mathbf{x}}(\varphi)| .
$$

Let $\mathbf{p}_{1}, \mathbf{p}_{2} \in \mathbb{S}(R)$ have coprime factorizations

$$
\mathbf{p}_{1}=\frac{\mathbf{n}_{1}}{\mathbf{d}_{1}} \text { and } \mathbf{p}_{2}=\frac{\mathbf{n}_{2}}{\mathbf{d}_{2}}
$$

Then the chordal pointwise distance function $\kappa_{\mathbf{p}_{1}, \mathbf{p}_{2}}$ is

$$
\kappa_{\mathbf{p}_{1}, \mathbf{p}_{2}}(\varphi):=\frac{\left|\widehat{\mathbf{n}_{1}}(\varphi) \widehat{\mathbf{d}_{2}}(\varphi)-\widehat{\mathbf{n}_{2}}(\varphi) \widehat{\mathbf{d}_{1}}(\varphi)\right|}{\sqrt{\left|\widehat{\mathbf{n}_{1}}(\varphi)\right|^{2}+\left|\widehat{\mathbf{d}_{1}}(\varphi)\right|^{2}} \sqrt{\left|\widehat{\mathbf{n}_{2}}(\varphi)\right|^{2}+\left|\widehat{\mathbf{d}_{2}}(\varphi)\right|^{2}}}, \quad \varphi \in M(S) .
$$

The function $\kappa$ given by the above expression is well-defined, that is, it does not depend on the choice of coprime factorizations for each of the plants; see [13]. 
Definition 4.2. For $\mathbf{p}_{1}, \mathbf{p}_{2} \in \mathbb{S}$, with coprime factorizations

we define

$$
\mathbf{p}_{1}=\frac{\mathbf{n}_{1}}{\mathbf{d}_{1}}, \quad \mathbf{p}_{2}=\frac{\mathbf{n}_{2}}{\mathbf{d}_{2}},
$$

$$
d_{c, r}\left(\mathbf{p}_{1}, \mathbf{p}_{2}\right):= \begin{cases}\left\|\kappa_{\mathbf{p}_{1}, \mathbf{p}_{2}}\right\|_{S, \infty} & \text { if } \mathbf{n}_{1}^{*} \mathbf{n}_{2}+\mathbf{d}_{1}^{*} \mathbf{d}_{2} \in \operatorname{inv} S \text { and } \iota\left(\mathbf{n}_{1}^{*} \mathbf{n}_{2}+\mathbf{d}_{1}^{*} \mathbf{d}_{2}\right)=\circ \\ 1 & \text { otherwise }\end{cases}
$$

$d_{c, r}$ given by (4.1) is well-defined, and it is bounded above by 1 . It is also clear that if the two plants have normalized coprime factorizations, that is, if also

$$
\mathbf{n}_{1}^{*} \mathbf{n}_{1}+\mathbf{d}_{1}^{*} \mathbf{d}_{1}=1=\mathbf{n}_{2}^{*} \mathbf{n}_{2}+\mathbf{d}_{2}^{*} \mathbf{d}_{2},
$$

then it is clear that $d_{c, r}\left(\mathbf{p}_{1}, \mathbf{p}_{2}\right)=d_{\nu}\left(\mathbf{p}_{1}, \mathbf{p}_{2}\right)$, where $d_{\nu}$ is the extension of the $\nu$-metric defined in [1]. We note however, that $d_{\nu}$ was defined on the set $\mathbb{S}_{n}$ of elements in the field of fractions possessing a normalized coprime factorization, and for some rings $R$ (for example $R=A(\mathbb{D}$ ), the disk algebra; see [14]), it may be the case that $\mathbb{S}_{n} \subsetneq \mathbb{S}$.

Our first main result is the following:

Theorem 4.3. $d_{c, r}$ is a metric on $\mathbb{S}$.

We will also show that stabilizability is a robust property of the plant and give the quantitative version of this in Theorem 4.5 below. But first, we have the following definition of the stability margin.

Definition 4.4. Given $\mathbf{p} \in \mathbb{F}(R)$, we say that $\mathbf{c} \in \mathbb{F}(R)$ stabilizes $\mathbf{p}$ if

$$
\mathbf{H}(\mathbf{p}, \mathbf{c}):=\left[\begin{array}{cc}
\frac{-\mathbf{p c}}{1-\mathbf{p c}} & \frac{\mathbf{p}}{1-\mathbf{p c}} \\
\frac{-\mathbf{c}}{1-\mathbf{p c}} & \frac{1}{1-\mathbf{p c}}
\end{array}\right] \in R^{2 \times 2} .
$$

We define the stability margin $\mu_{\mathbf{p}, \mathbf{c}}$ of $\mathbf{p}, \mathbf{c} \in \mathbb{F}(R)$ by

$$
\mu_{\mathbf{p}, \mathbf{c}}=\left\{\begin{array}{cl}
\frac{1}{\|\mathbf{H}(\mathbf{p}, \mathbf{c})\|_{S, \infty}} & \text { if } \mathbf{c} \text { stabilizes } \mathbf{p} \\
0 & \text { otherwise }
\end{array}\right.
$$

Here for a matrix $M \in S^{p \times m}$ with entries from $S$, we set

$$
\|M\|_{S, \infty}=\sup _{\varphi \in M(S)}|\widehat{M}(\varphi)|
$$

where $\widehat{M}$ denotes the matrix obtained by taking entrywise Gelfand transforms, and $|\cdot|$ denotes the induced operator norm when $\mathbb{C}^{p}, \mathbb{C}^{m}$ are equipped with the usual Euclidean norms.

Theorem 4.5. If $\mathbf{p}, \mathbf{p}_{0}, \mathbf{c} \in \mathbb{S}$, then $\mu_{\mathbf{p}, \mathbf{c}} \geq \mu_{\mathbf{p}_{0}, \mathbf{c}}-d_{c, r}\left(\mathbf{p}, \mathbf{p}_{0}\right)$.

In order to show Theorem 4.5, the following fact will be useful.

Lemma 4.6. If $\mathbf{p}, \mathbf{c} \in \mathbb{S}$ have coprime factorizations $\mathbf{p}=\frac{\mathbf{n}_{p}}{\mathbf{d}_{p}}$ and $\mathbf{c}=\frac{\mathbf{n}_{c}}{\mathbf{d}_{c}}$, then

$$
\mu_{\mathbf{p}, \mathbf{c}}=\inf _{\varphi \in M(S)} \frac{\left|\widehat{\mathbf{n}}_{p}(\varphi) \widehat{\mathbf{n}}_{c}(\varphi)-\widehat{\mathbf{d}}_{p}(\varphi) \widehat{\mathbf{d}}_{c}(\varphi)\right|}{\sqrt{\left|\widehat{\mathbf{n}}_{p}(\varphi)\right|^{2}+\left|\widehat{\mathbf{d}}_{p}(\varphi)\right|^{2}} \sqrt{\left|\widehat{\mathbf{n}}_{c}(\varphi)\right|^{2}+\left|\widehat{\mathbf{d}}_{c}(\varphi)\right|^{2}}} .
$$


Proof. The claim follows from the observations that

$$
\mathbf{H}(\mathbf{p}, \mathbf{c})=\frac{1}{\mathbf{d}_{p} \mathbf{d}_{c}-\mathbf{n}_{p} \mathbf{n}_{c}}\left[\begin{array}{l}
\mathbf{n}_{p} \\
\mathbf{d}_{p}
\end{array}\right]\left[\begin{array}{ll}
-\mathbf{n}_{c} & \mathbf{d}_{c}
\end{array}\right]
$$

and

$$
\mathbf{I}\left[\begin{array}{c}
\widehat{\mathbf{n}}_{p}(\varphi) \\
\widehat{\mathbf{d}}_{p}(\varphi)
\end{array}\right]\left[\begin{array}{ll}
-\widehat{\mathbf{n}}_{c}(\varphi) & \widehat{\mathbf{d}}_{c}(\varphi)
\end{array}\right] \mathbf{I}=\sqrt{\left|\widehat{\mathbf{n}}_{p}(\varphi)\right|^{2}+\left|\widehat{\mathbf{d}}_{p}(\varphi)\right|^{2}} \sqrt{\left|\widehat{\mathbf{n}}_{c}(\varphi)\right|^{2}+\left|\widehat{\mathbf{d}}_{c}(\varphi)\right|^{2}},
$$

for $\varphi \in M(S)$.

It follows from the above that $\mu_{\mathbf{p}, \mathbf{c}} \leq 1$. Also, $\mu_{\mathbf{p}, \mathbf{c}}=\mu_{\mathbf{c}, \mathbf{p}}$.

Proposition 4.7. Let $\mathbf{p}, \mathbf{c} \in \mathbb{S}$ have coprime factorizations

$$
\mathbf{p}=\frac{\mathbf{n}_{p}}{\mathbf{d}_{p}} \text { and } \mathbf{c}=\frac{\mathbf{n}_{c}}{\mathbf{d}_{c}} .
$$

Then the following are equivalent:

(1) c stabilizes $\mathbf{p}$.

(2) $\mathbf{n}_{p} \mathbf{n}_{c}-\mathbf{d}_{p} \mathbf{d}_{c} \in \operatorname{inv} S$ and $\iota\left(\mathbf{n}_{p} \mathbf{n}_{c}-\mathbf{d}_{p} \mathbf{d}_{c}\right)=0$.

Proof. Suppose that c stabilizes $\mathbf{p}$. Then it can be seen that $\mathbf{n}_{p} \mathbf{n}_{c}-\mathbf{d}_{p} \mathbf{d}_{c}$ belongs to inv $R$. It follows from (I4) that $\mathbf{n}_{p} \mathbf{n}_{c}-\mathbf{d}_{p} \mathbf{d}_{c} \in \operatorname{inv} S$ and that $\iota\left(\mathbf{n}_{p} \mathbf{n}_{c}-\mathbf{d}_{p} \mathbf{d}_{c}\right)=0$.

On the other hand, if $\mathbf{n}_{p} \mathbf{n}_{c}-\mathbf{d}_{p} \mathbf{d}_{c} \in$ inv $S$ and $\iota\left(\mathbf{n}_{p} \mathbf{n}_{c}-\mathbf{d}_{p} \mathbf{d}_{c}\right)=\circ$, then again by (I4), we obtain that $\mathbf{n}_{p} \mathbf{n}_{c}-\mathbf{d}_{p} \mathbf{d}_{c} \in$ inv $R$. It follows from here that $\mathbf{H}(\mathbf{p}, \mathbf{c})$ has each entry in $R$.

The following elementary fact will be used often in our proofs.

Lemma 4.8. For complex numbers $a, b, \alpha, \beta$, such that $|a|^{2}+|b|^{2}>0$ and $|\alpha|^{2}+|\beta|^{2}>0$ there holds that

$$
1-\frac{|a \beta-b \alpha|^{2}}{\left(|a|^{2}+|b|^{2}\right)\left(|\alpha|^{2}+|\beta|^{2}\right)}=\frac{|a \bar{\alpha}+b \bar{\beta}|^{2}}{\left(|a|^{2}+|b|^{2}\right)\left(|\alpha|^{2}+|\beta|^{2}\right)} .
$$

The proof of Theorems 4.3 and 4.5 are analogous to the proofs given in [16] (in the case when $R$ is the ring of rational functions bounded in the right half complex plane) and the proofs given in [1] (in an abstract setting). However, Theorems 4.3 and 4.5 are not automatic, and do not follow from [1], since we consider a more general setting than the one considered in [1] (merely coprime factorizations versus normalized coprime factorizations).

$$
\text { 5. } d_{c, r} \text { IS A METRIC }
$$

Proof of Theorem 4.3. The fact that $d_{c, r}$ is nonnegative is trivial. Also, if $d_{c, r}\left(\mathbf{p}_{1}, \mathbf{p}_{2}\right)=0$, then it is clear that $\mathbf{p}_{1}=\mathbf{p}_{2}$. Symmetry is also easy to check. We only check the triangle inequality. Suppose that $\mathbf{p}_{1}, \mathbf{p}_{2}, \mathbf{p}_{0} \in \mathbb{S}$. We want to show that

$$
d_{c, r}\left(\mathbf{p}_{1}, \mathbf{p}_{2}\right) \leq d_{c, r}\left(\mathbf{p}_{1}, \mathbf{p}_{0}\right)+d_{c, r}\left(\mathbf{p}_{0}, \mathbf{p}_{2}\right) \text {. }
$$

Since $d_{c, r}$ is bounded above by 1 , this inequality is trivially satisfied if either $d_{c, r}\left(\mathbf{p}_{1}, \mathbf{p}_{0}\right)=1$ or $d_{c, r}\left(\mathbf{p}_{0}, \mathbf{p}_{2}\right)=1$. So we assume that $d_{c, r}\left(\mathbf{p}_{1}, \mathbf{p}_{0}\right)<1$ and $d_{c, r}\left(\mathbf{p}_{0}, \mathbf{p}_{2}\right)<1$. Let

$$
\mathbf{p}_{0}=\frac{\mathbf{n}_{0}}{\mathbf{d}_{0}}, \quad \mathbf{p}_{1}=\frac{\mathbf{n}_{1}}{\mathbf{d}_{1}}, \quad \mathbf{p}_{2}=\frac{\mathbf{n}_{2}}{\mathbf{d}_{2}},
$$

be coprime factorizations. If $\mathbf{n}_{1}^{*} \mathbf{n}_{2}+\mathbf{d}_{1}^{*} \mathbf{d}_{2} \in \operatorname{inv} S$ and if $\iota\left(\mathbf{n}_{1}^{*} \mathbf{n}_{2}+\mathbf{d}_{1}^{*} \mathbf{d}_{2}\right)=\circ$, then the inequality follows from the triangle inequality in $\mathbb{R}^{3}$, that is from the triangle inequality for the generalized chordal metric; see [13]. 
So we will assume that $\neg\left[\mathbf{n}_{1}^{*} \mathbf{n}_{2}+\mathbf{d}_{1}^{*} \mathbf{d}_{2} \in\right.$ inv $S$ and $\left.\iota\left(\mathbf{n}_{1}^{*} \mathbf{n}_{2}+\mathbf{d}_{1}^{*} \mathbf{d}_{2}\right)=0\right]$. Let

$$
\begin{aligned}
& A:=\underbrace{\left(\mathbf{n}_{1}^{*} \mathbf{n}_{0}+\mathbf{d}_{1}^{*} \mathbf{d}_{0}\right)}_{\in \text { inv } S} \underbrace{\left(\mathbf{n}_{0}^{*} \mathbf{n}_{2}+\mathbf{d}_{0}^{*} \mathbf{d}_{2}\right)}_{\in \operatorname{inv} S} \in \operatorname{inv} S, \\
& B:=\left(-\mathbf{n}_{1}^{*} \mathbf{d}_{0}^{*}+\mathbf{d}_{1}^{*} \mathbf{n}_{0}^{*}\right)\left(-\mathbf{d}_{0} \mathbf{n}_{2}+\mathbf{n}_{0} \mathbf{d}_{2}\right) .
\end{aligned}
$$

Suppose that $\left\|A^{-1} B\right\|_{S, \infty}<1$. Then

$$
\left(\mathbf{n}_{1}^{*} \mathbf{n}_{2}+\mathbf{d}_{1}^{*} \mathbf{d}_{2}\right) \cdot \underbrace{\left(\left|\mathbf{n}_{0}\right|^{2}+\left|\mathbf{d}_{0}\right|^{2}\right)}_{\in \text { inv } S}=A+B=\underbrace{A\left(1+A^{-1} B\right)}_{\in \text { inv } S},
$$

and so $\mathbf{n}_{1}^{*} \mathbf{n}_{2}+\mathbf{d}_{1}^{*} \mathbf{d}_{2} \in \operatorname{inv} S$. Let $H:[0,1] \rightarrow \operatorname{inv} S$ be defined by $H(t)=A\left(1+t A^{-1} B\right)$, $t \in[0,1]$. By the homotopic invariance of the index, $\iota(A)=\iota(H(0))=\iota(H(1))=\iota(A+B)$. But $\iota(A)=\circ+\circ=\circ$. And using (I5), $\iota(A+B)=\iota\left(\mathbf{n}_{1}^{*} \mathbf{n}_{2}+\mathbf{d}_{1}^{*} \mathbf{d}_{2}\right)+\circ$. Thus $\iota\left(\mathbf{n}_{1}^{*} \mathbf{n}_{2}+\mathbf{d}_{1}^{*} \mathbf{d}_{2}\right)=\circ$, a contradiction.

So it can't be the case that $\left\|A^{-1} B\right\|_{S, \infty}<1$. Since $M(S)$ is compact, it follows from here that there exists a $\varphi_{0} \in M(S)$ such that $\left|\widehat{B}\left(\varphi_{0}\right)\right| \geq\left|\widehat{A}\left(\varphi_{0}\right)\right|$. Using Lemma 4.8, we obtain that

$$
\begin{aligned}
& \left(1-\left(\kappa_{\mathbf{p}_{0}, \mathbf{p}_{1}}\left(\varphi_{0}\right)\right)^{2}\right)\left(1-\left(\kappa_{\mathbf{p}_{0}, \mathbf{p}_{2}}\left(\varphi_{0}\right)\right)^{2}\right) \\
= & \frac{\left|\widehat{A}\left(\varphi_{0}\right)\right|^{2}}{\left(\left|\widehat{\mathbf{n}}_{0}\left(\varphi_{0}\right)\right|^{2}+\left|\widehat{\mathbf{d}}_{0}\left(\varphi_{0}\right)\right|^{2}\right)^{2}\left(\left|\widehat{\mathbf{n}}_{1}\left(\varphi_{0}\right)\right|^{2}+\left|\widehat{\mathbf{d}}_{1}\left(\varphi_{0}\right)\right|^{2}\right)\left(\left|\widehat{\mathbf{n}}_{2}\left(\varphi_{0}\right)\right|^{2}+\left|\widehat{\mathbf{d}}_{2}\left(\varphi_{0}\right)\right|^{2}\right)} \\
\leq & \frac{\left|\widehat{B}\left(\varphi_{0}\right)\right|^{2}}{\left(\left|\widehat{\mathbf{n}}_{0}\left(\varphi_{0}\right)\right|^{2}+\left|\widehat{\mathbf{d}}_{0}\left(\varphi_{0}\right)\right|^{2}\right)^{2}\left(\left|\widehat{\mathbf{n}}_{1}\left(\varphi_{0}\right)\right|^{2}+\left|\widehat{\mathbf{d}}_{1}\left(\varphi_{0}\right)\right|^{2}\right)\left(\left|\widehat{\mathbf{n}}_{2}\left(\varphi_{0}\right)\right|^{2}+\left|\widehat{\mathbf{d}}_{2}\left(\varphi_{0}\right)\right|^{2}\right)} \\
= & \left(\kappa_{\mathbf{p}_{0}, \mathbf{p}_{1}}\left(\varphi_{0}\right)\right)^{2} \cdot\left(\left(\kappa_{\mathbf{p}_{0}, \mathbf{p}_{2}}\left(\varphi_{0}\right)\right)^{2} \cdot\right.
\end{aligned}
$$

Upon rearranging, we obtain $\left(\kappa_{\mathbf{p}_{0}, \mathbf{p}_{1}}\left(\varphi_{0}\right)\right)^{2}+\left(\kappa_{\mathbf{p}_{0}, \mathbf{p}_{2}}\left(\varphi_{0}\right)\right)^{2} \geq 1$, and so

$$
\begin{aligned}
\left(d_{c, r}\left(\mathbf{p}_{0}, \mathbf{p}_{1}\right)+d_{c, r}\left(\mathbf{p}_{0}, \mathbf{p}_{2}\right)\right)^{2} & =\left(\kappa_{\mathbf{p}_{0}, \mathbf{p}_{1}}\left(\varphi_{0}\right)+\kappa_{\mathbf{p}_{0}, \mathbf{p}_{2}}\left(\varphi_{0}\right)\right)^{2} \\
& \geq\left(\kappa_{\mathbf{p}_{0}, \mathbf{p}_{1}}\left(\varphi_{0}\right)\right)^{2}+\left(\kappa_{\mathbf{p}_{0}, \mathbf{p}_{2}}\left(\varphi_{0}\right)\right)^{2} \\
& \geq 1=\left(d_{c, r}\left(\mathbf{p}_{1}, \mathbf{p}_{2}\right)\right)^{2} .
\end{aligned}
$$

This completes the proof of the triangle inequality, and also the proof of Theorem 4.3 .

\section{Stabilizability is a Robust property}

Proof of Theorem 4.5. If $d_{c, r}\left(\mathbf{p}, \mathbf{p}_{0}\right) \geq \mu_{\mathbf{p}_{0}, \mathbf{c}}$, then trivially we have

$$
\mu_{\mathbf{p}, \mathbf{c}} \geq 0 \geq \mu_{\mathbf{p}_{0}, \mathbf{c}}-d_{c, r}\left(\mathbf{p}, \mathbf{p}_{0}\right) .
$$

So let us suppose that $d_{c, r}\left(\mathbf{p}, \mathbf{p}_{0}\right)<\mu_{\mathbf{p}_{0}, \mathbf{c}}$. Since $\mu_{\mathbf{p}_{0}, \mathbf{c}} \leq 1$, it follows that $d_{c, r}\left(\mathbf{p}, \mathbf{p}_{0}\right)<1$. Also, $\mu_{\mathbf{p}_{0}, \mathbf{c}} \neq 0$, since otherwise we would have $d_{c, r}\left(\mathbf{p}, \mathbf{p}_{0}\right)<0$, which is impossible. So 
$\mu_{\mathbf{p}_{0}, \mathbf{c}}>0$. Hence $\mathbf{c}$ stabilizes $\mathbf{p}_{0}$. We have

$$
\begin{aligned}
d_{c, r}\left(\mathbf{p}, \mathbf{p}_{0}\right) & =\sup _{\varphi \in M(S)} \frac{\left|\widehat{\mathbf{n}}_{p}(\varphi) \widehat{\mathbf{d}}_{p, 0}(\varphi)-\widehat{\mathbf{n}}_{p}(\varphi) \widehat{\mathbf{d}}_{p, 0}(\varphi)\right|}{\sqrt{\left|\widehat{\mathbf{n}}_{p}(\varphi)\right|^{2}+\left|\widehat{\mathbf{d}}_{p}(\varphi)\right|^{2}} \sqrt{\left|\widehat{\mathbf{n}}_{p, 0}(\varphi)\right|^{2}+\left|\widehat{\mathbf{d}}_{p, 0}(\varphi)\right|^{2}}} \\
& <\inf _{\varphi \in M(S)} \frac{\left|\widehat{\mathbf{n}}_{p, 0}(\varphi) \widehat{\mathbf{n}}_{c}(\varphi)-\widehat{\mathbf{d}}_{p, 0}(\varphi) \widehat{\mathbf{d}}_{c}(\varphi)\right|}{\sqrt{\left|\widehat{\mathbf{n}}_{p, 0}(\varphi)\right|^{2}+\left|\widehat{\mathbf{d}}_{p, 0}(\varphi)\right|^{2}} \sqrt{\left|\widehat{\mathbf{n}}_{c}(\varphi)\right|^{2}+\left|\widehat{\mathbf{d}}_{c}(\varphi)\right|^{2}}} \\
& =\mu_{\mathbf{p}_{0}, \mathbf{c}} .
\end{aligned}
$$

So for all $\varphi \in M(S)$,

$$
\begin{aligned}
& \frac{\left|\widehat{\mathbf{n}}_{p}(\varphi) \widehat{\mathbf{d}}_{p, 0}(\varphi)-\widehat{\mathbf{n}}_{p}(\varphi) \widehat{\mathbf{d}}_{p, 0}(\varphi)\right|}{\sqrt{\left|\widehat{\mathbf{n}}_{p}(\varphi)\right|^{2}+\left|\widehat{\mathbf{d}}_{p}(\varphi)\right|^{2}} \sqrt{\left|\widehat{\mathbf{n}}_{p, 0}(\varphi)\right|^{2}+\left|\widehat{\mathbf{d}}_{p, 0}(\varphi)\right|^{2}}} \\
< & \frac{\left|\widehat{\mathbf{n}}_{p, 0}(\varphi) \widehat{\mathbf{n}}_{c}(\varphi)-\widehat{\mathbf{d}}_{p, 0}(\varphi) \widehat{\mathbf{d}}_{c}(\varphi)\right|}{\sqrt{\left|\widehat{\mathbf{n}}_{p, 0}(\varphi)\right|^{2}+\left|\widehat{\mathbf{d}}_{p, 0}(\varphi)\right|^{2}} \sqrt{\left|\widehat{\mathbf{n}}_{c}(\varphi)\right|^{2}+\left|\widehat{\mathbf{d}}_{c}(\varphi)\right|^{2}}}
\end{aligned}
$$

Using the elementary result in Lemma 4.8, we obtain

$$
\begin{aligned}
& \frac{\left|\left(\widehat{\mathbf{d}}_{p, 0}(\varphi)\right)^{*} \widehat{\mathbf{n}}_{c}(\varphi)+\left(\widehat{\mathbf{n}}_{p, 0}(\varphi)\right)^{*} \widehat{\mathbf{d}}_{c}(\varphi)\right|}{\sqrt{\left|\widehat{\mathbf{n}}_{c}(\varphi)\right|^{2}+\left|\widehat{\mathbf{d}}_{c}(\varphi)\right|^{2}} \sqrt{\left|\widehat{\mathbf{n}}_{p, 0}(\varphi)\right|^{2}+\left|\widehat{\mathbf{d}}_{p, 0}(\varphi)\right|^{2}}} \\
< & \frac{\left|\left(\widehat{\mathbf{n}}_{p, 0}(\varphi)\right)^{*} \widehat{\mathbf{n}}_{p}(\varphi)+\left(\widehat{\mathbf{d}}_{p, 0}(\varphi)\right)^{*} \widehat{\mathbf{d}}_{p}(\varphi)\right|}{\sqrt{\left|\widehat{\mathbf{n}}_{p, 0}(\varphi)\right|^{2}+\left|\widehat{\mathbf{d}}_{p, 0}(\varphi)\right|^{2}} \sqrt{\left|\widehat{\mathbf{n}}_{p}(\varphi)\right|^{2}+\left|\widehat{\mathbf{d}}_{p}(\varphi)\right|^{2}}}
\end{aligned}
$$

Since all quantities in the inequalities (6.1), (6.2) are positive, we may multiply them to obtain

$$
\begin{array}{r}
\left|\left(\widehat{\mathbf{d}}_{p, 0}(\varphi)\right)^{*} \widehat{\mathbf{n}}_{c}(\varphi)+\left(\widehat{\mathbf{n}}_{p, 0}(\varphi)\right)^{*} \widehat{\mathbf{d}}_{c}(\varphi)\right|\left|\widehat{\mathbf{n}}_{p}(\varphi) \widehat{\mathbf{d}}_{p, 0}(\varphi)-\widehat{\mathbf{n}}_{p}(\varphi) \widehat{\mathbf{d}}_{p, 0}(\varphi)\right| \\
<\left|\left(\widehat{\mathbf{n}}_{p, 0}(\varphi)\right)^{*} \widehat{\mathbf{n}}_{p}(\varphi)+\left(\widehat{\mathbf{d}}_{p, 0}(\varphi)\right)^{*} \widehat{\mathbf{d}}_{p}(\varphi)\right|\left|\widehat{\mathbf{n}}_{p, 0}(\varphi) \widehat{\mathbf{n}}_{c}(\varphi)-\widehat{\mathbf{d}}_{p, 0}(\varphi) \widehat{\mathbf{d}}_{c}(\varphi)\right|
\end{array}
$$

Define

$$
\begin{aligned}
& A:=\underbrace{\left(\mathbf{n}_{p, 0}^{*} \mathbf{n}_{p}+\mathbf{d}_{p, 0}^{*} \mathbf{d}_{p}\right)}_{\in \operatorname{inv} S \text { as } d_{c, r}\left(\mathbf{p}, \mathbf{p}_{0}\right)<1} \cdot \underbrace{\left(-\mathbf{n}_{c} \mathbf{n}_{p, 0}+\mathbf{d}_{c} \mathbf{d}_{p, 0}\right)}_{\in \operatorname{inv} S \text { as c stabilizes } \mathbf{p}_{0}} \in \operatorname{inv} S, \\
& B:=\left(-\mathbf{n}_{p} \mathbf{d}_{p, 0}+\mathbf{d}_{p, 0} \mathbf{d}_{p}\right) \cdot\left(\mathbf{d}_{p, 0}^{*} \mathbf{n}_{c}+\mathbf{n}_{p, 0}^{*} \mathbf{d}_{c}\right) .
\end{aligned}
$$

From (6.3), we know that $\left\|A^{-1} B\right\|_{S, \infty}<1$. Since

$$
\left(\mathbf{n}_{p} \mathbf{n}_{c}-\mathbf{d}_{p} \mathbf{d}_{c}\right) \underbrace{\left(\left|\mathbf{n}_{p, 0}\right|^{2}+\left|\mathbf{d}_{p, 0}\right|^{2}\right)}_{\in \text { inv } S}=A+B=\underbrace{A\left(1+A^{-1} B\right)}_{\text {inv } S},
$$

it follows that $\mathbf{n}_{p} \mathbf{n}_{c}-\mathbf{d}_{p} \mathbf{d}_{c} \in \operatorname{inv} S$. Define the map $H:[0,1] \rightarrow \operatorname{inv} S$ by $H(t)=A(1+$ $\left.t A^{-1} B\right), t \in[0,1]$. By Proposition 4.1, $\iota(H(0))=\iota(H(1))$. But $\iota(H(0))=\iota(A)=\circ+\circ=\circ$, and

$$
\iota(H(1))=\iota\left(\mathbf{n}_{p} \mathbf{n}_{c}-\mathbf{d}_{p} \mathbf{d}_{c}\right)+\circ=\iota\left(\mathbf{n}_{p} \mathbf{n}_{c}-\mathbf{d}_{p} \mathbf{d}_{c}\right) .
$$

Consequently, $\iota\left(\mathbf{n}_{p} \mathbf{n}_{c}-\mathbf{d}_{p} \mathbf{d}_{c}\right)=$ 。. It follows from Proposition 4.7 that $\mathbf{c}$ stabilizes $\mathbf{p}$. 
It is easy to check that

$$
\begin{aligned}
& \left(\mathbf{n}_{p} \mathbf{n}_{c}-\mathbf{d}_{p} \mathbf{d}_{c}\right)\left(\left|\mathbf{n}_{p, 0}\right|^{2}+\left|\mathbf{d}_{p, 0}\right|^{2}\right) \\
= & \left(\mathbf{n}_{p, 0}^{*} \mathbf{n}_{p}+\mathbf{d}_{p, 0}^{*} \mathbf{d}_{p}\right)\left(\mathbf{n}_{c} \mathbf{n}_{p, 0}-\mathbf{d}_{c} \mathbf{d}_{p, 0}\right)+\left(\mathbf{n}_{p} \mathbf{d}_{p, 0}-\mathbf{n}_{p, 0} \mathbf{d}_{p}\right)\left(\mathbf{n}_{c} \mathbf{d}_{p, 0}^{*}+\mathbf{n}_{p, 0}^{*} \mathbf{d}_{c}\right) .
\end{aligned}
$$

Thus for $\varphi \in M(S)$,

$$
\begin{aligned}
& \frac{\left|\widehat{\mathbf{n}}_{p}(\varphi) \widehat{\mathbf{n}}_{c}(\varphi)-\widehat{\mathbf{d}}_{p}(\varphi) \widehat{\mathbf{d}}_{c}(\varphi)\right|}{\sqrt{\left|\widehat{\mathbf{n}}_{p}(\varphi)\right|^{2}+\left|\widehat{\mathbf{d}}_{p}(\varphi)\right|^{2}} \sqrt{\left|\widehat{\mathbf{n}}_{c}(\varphi)\right|^{2}+\left|\widehat{\mathbf{d}}_{c}(\varphi)\right|^{2}}} \\
= & \mid \frac{\left(\widehat{\mathbf{n}}_{p, 0}^{*}(\varphi) \widehat{\mathbf{n}}_{p}(\varphi)+\widehat{\mathbf{d}}_{p, 0}^{*}(\varphi) \widehat{\mathbf{d}}_{p}(\varphi)\right)\left(\widehat{\mathbf{n}}_{c}(\varphi) \widehat{\mathbf{n}}_{p, 0}(\varphi)-\widehat{\mathbf{d}}_{c}(\varphi) \widehat{\mathbf{d}}_{p, 0}(\varphi)\right)}{\sqrt{\left|\widehat{\mathbf{n}}_{p}(\varphi)\right|^{2}+\left|\widehat{\mathbf{d}}_{p}(\varphi)\right|^{2}} \sqrt{\left|\widehat{\mathbf{n}}_{c}(\varphi)\right|^{2}+\left|\widehat{\mathbf{d}}_{c}(\varphi)\right|^{2}}\left(\left|\widehat{\mathbf{n}}_{p, 0}(\varphi)\right|^{2}+\left|\widehat{\mathbf{d}}_{p, 0}(\varphi)\right|^{2}\right)} \\
& +\frac{\left(\widehat{\mathbf{n}}_{p}(\varphi) \widehat{\mathbf{d}}_{p, 0}(\varphi)-\widehat{\mathbf{n}}_{p, 0}(\varphi) \widehat{\mathbf{d}}_{p}(\varphi)\right)\left(\widehat{\mathbf{n}}_{c}(\varphi) \widehat{\mathbf{d}}_{p, 0}^{*}(\varphi)+\widehat{\mathbf{n}}_{p, 0}^{*}(\varphi) \widehat{\mathbf{d}}_{c}(\varphi)\right)}{\sqrt{\left|\widehat{\mathbf{n}}_{p}(\varphi)\right|^{2}+\left|\widehat{\mathbf{d}}_{p}(\varphi)\right|^{2}} \sqrt{\left|\widehat{\mathbf{n}}_{c}(\varphi)\right|^{2}+\left|\widehat{\mathbf{d}}_{c}(\varphi)\right|^{2}}\left(\left|\widehat{\mathbf{n}}_{p, 0}(\varphi)\right|^{2}+\left|\widehat{\mathbf{d}}_{p, 0}(\varphi)\right|^{2}\right) \mid} \\
\geq & (\sin \alpha) \cdot(\cos \beta)-(\cos \alpha) \cdot(\sin \beta)=\sin (\alpha-\beta),
\end{aligned}
$$

where $\alpha, \beta \in[0, \pi / 2]$ are such that

$$
\begin{aligned}
\sin \alpha & =\frac{\left|-\widehat{\mathbf{n}}_{p, 0}(\varphi) \widehat{\mathbf{n}}_{c}(\varphi)+\widehat{\mathbf{d}}_{p, 0}(\varphi) \widehat{\mathbf{d}}_{c}(\varphi)\right|}{\sqrt{\left|\widehat{\mathbf{n}}_{c}(\varphi)\right|^{2}+\left|\widehat{\mathbf{d}}_{c}(\varphi)\right|^{2}} \sqrt{\left|\widehat{\mathbf{n}}_{p, 0}(\varphi)\right|^{2}+\left|\widehat{\mathbf{d}}_{p, 0}(\varphi)\right|^{2}}}, \\
\sin \beta & =\frac{\left|-\widehat{\mathbf{n}}_{p}(\varphi) \widehat{\mathbf{d}}_{p, 0}(\varphi)+\widehat{\mathbf{n}}_{p, 0}(\varphi) \widehat{\mathbf{d}}_{p}(\varphi)\right|}{\sqrt{\left|\widehat{\mathbf{n}}_{p}(\varphi)\right|^{2}+\left|\widehat{\mathbf{d}}_{p}(\varphi)\right|^{2}} \sqrt{\left|\widehat{\mathbf{n}}_{p, 0}(\varphi)\right|^{2}+\left|\widehat{\mathbf{d}}_{p, 0}(\varphi)\right|^{2}}}
\end{aligned}
$$

Then

$$
\begin{aligned}
\cos \alpha & =\frac{\left|\widehat{\mathbf{n}}_{c}(\varphi) \widehat{\mathbf{d}}_{p, 0}^{*}(\varphi)+\widehat{\mathbf{d}}_{c}(\varphi) \widehat{\mathbf{n}}_{p, 0}^{*}(\varphi)\right|}{\sqrt{\left|\widehat{\mathbf{n}}_{c}(\varphi)\right|^{2}+\left|\widehat{\mathbf{d}}_{c}(\varphi)\right|^{2}} \sqrt{\left|\widehat{\mathbf{n}}_{p, 0}(\varphi)\right|^{2}+\left|\widehat{\mathbf{d}}_{p, 0}(\varphi)\right|^{2}}}, \\
\cos \beta & =\frac{\left|\widehat{\mathbf{n}}_{p}(\varphi) \widehat{\mathbf{n}}_{p, 0}^{*}(\varphi)+\widehat{\mathbf{d}}_{p, 0}(\varphi) \widehat{\mathbf{d}}_{p, 0}^{*}(\varphi)\right|}{\sqrt{\left|\widehat{\mathbf{n}}_{p}(\varphi)\right|^{2}+\left|\widehat{\mathbf{d}}_{p}(\varphi)\right|^{2}} \sqrt{\left|\widehat{\mathbf{n}}_{p, 0}(\varphi)\right|^{2}+\left|\widehat{\mathbf{d}}_{p, 0}(\varphi)\right|^{2}}} .
\end{aligned}
$$

Since $\sin ^{-1}:[0,1] \rightarrow[0, \pi / 2]$ is increasing, we obtain for $\varphi \in M(S)$,

$$
\sin ^{-1} \frac{\left|\widehat{\mathbf{n}}_{p}(\varphi) \widehat{\mathbf{n}}_{c}(\varphi)-\widehat{\mathbf{d}}_{p}(\varphi) \widehat{\mathbf{d}}_{c}(\varphi)\right|}{\sqrt{\left|\widehat{\mathbf{n}}_{p}(\varphi)\right|^{2}+\left|\widehat{\mathbf{d}}_{p}(\varphi)\right|^{2}} \sqrt{\left|\widehat{\mathbf{n}}_{c}(\varphi)\right|^{2}+\left|\widehat{\mathbf{d}}_{c}(\varphi)\right|^{2}}} \geq \alpha-\beta
$$


But

$$
\begin{aligned}
\alpha-\beta= & \sin ^{-1} \frac{\left|-\widehat{\mathbf{n}}_{p, 0}(\varphi) \widehat{\mathbf{n}}_{c}(\varphi)+\widehat{\mathbf{d}}_{p, 0}(\varphi) \widehat{\mathbf{d}}_{c}(\varphi)\right|}{\sqrt{\left|\widehat{\mathbf{n}}_{c}(\varphi)\right|^{2}+\left|\widehat{\mathbf{d}}_{c}(\varphi)\right|^{2}} \sqrt{\left|\widehat{\mathbf{n}}_{p, 0}(\varphi)\right|^{2}+\left|\widehat{\mathbf{d}}_{p, 0}(\varphi)\right|^{2}}} \\
& -\sin ^{-1} \frac{\left|-\widehat{\mathbf{n}}_{p}(\varphi) \widehat{\mathbf{d}}_{p, 0}(\varphi)+\widehat{\mathbf{n}}_{p, 0}(\varphi) \widehat{\mathbf{d}}_{p}(\varphi)\right|}{\sqrt{\left|\widehat{\mathbf{n}}_{p}(\varphi)\right|^{2}+\left|\widehat{\mathbf{d}}_{p}(\varphi)\right|^{2}} \sqrt{\left|\widehat{\mathbf{n}}_{p, 0}(\varphi)\right|^{2}+\left|\widehat{\mathbf{d}}_{p, 0}(\varphi)\right|^{2}}} \\
\geq & \sin ^{-1} \mu_{\mathbf{p}_{0}, \mathbf{c}}-\sin ^{-1} \frac{\left|-\widehat{\mathbf{n}}_{p}(\varphi) \widehat{\mathbf{d}}_{p, 0}(\varphi)+\widehat{\mathbf{n}}_{p, 0}(\varphi) \widehat{\mathbf{d}}_{p}(\varphi)\right|}{\sqrt{\left|\widehat{\mathbf{n}}_{p}(\varphi)\right|^{2}+\left|\widehat{\mathbf{d}}_{p}(\varphi)\right|^{2}} \sqrt{\left|\widehat{\mathbf{n}}_{p, 0}(\varphi)\right|^{2}+\left|\widehat{\mathbf{d}}_{p, 0}(\varphi)\right|^{2}}} \\
\geq & \sin ^{-1} \mu_{\mathbf{p}_{0}, \mathbf{c}}-\sin ^{-1} d_{c, r}\left(\mathbf{p}, \mathbf{p}_{0}\right) .
\end{aligned}
$$

Hence

$$
\sin ^{-1} \mu_{\mathbf{p}, \mathbf{c}} \geq \sin ^{-1} \mu_{\mathbf{p}_{0}, \mathbf{c}}-\sin ^{-1} d_{c, r}\left(\mathbf{p}, \mathbf{p}_{0}\right) .
$$

For $x, y, z \in[0,1]$, if $\sin ^{-1} x \leq \sin ^{-1} y+\sin ^{-1} z$, then by taking the cosine of both sides and using that $\cos$ is a decreasing function on $\left[0, \frac{\pi}{2}\right]$, we get $\sqrt{1-x^{2}} \geq \sqrt{1-y^{2}} \sqrt{1-z^{2}}-y z$, which in turn implies that $\left(\sqrt{1-x^{2}}+y z\right)^{2} \geq\left(1-y^{2}\right)\left(1-z^{2}\right)$. Hence

$$
x^{2} \leq y^{2}+z^{2}+2 y z \sqrt{1-x^{2}} \leq y^{2}+z^{2}+2 y z \cdot 1=(y+z)^{2},
$$

which gives finally that $x \leq y+z$. The claimed inequality now follows immediately from the inequality in (6.4) upon setting $x=\mu_{\mathbf{p}_{0}, \mathbf{c}}, y=d_{c, r}\left(\mathbf{p}_{0}, \mathbf{p}\right)$ and $z=\mu_{\mathbf{p}, \mathbf{c}}$.

\section{SPECIFIC InSTANCES OF $R$}

Table 7 below gives an overview of the choice of principal objects $S, G, \iota$ specific to choices of $R$ as the standard classes of stable transfer functions used in control theory.

\begin{tabular}{|c||c|c|c|}
\hline$R$ & $S$ & $G$ & $\iota$ \\
\hline \hline $\begin{array}{c}R H^{\infty} \\
A(\mathbb{D}), \\
\frac{W^{+}(\mathbb{D}),}{\left.L^{1[0, \infty}\right)+\mathbb{C},} \\
\cdots\end{array}$ & $C(\mathbb{T})$ & $\mathbb{Z}$ & $f \mapsto w(f)$ \\
\hline $\mathcal{A}_{+}$ & $C_{0}+A P$ & $\mathbb{R} \times \mathbb{Z}$ & $f=f_{0}+f_{A P} \mapsto\left(w_{\text {av }}\left(f_{A P}\right)+w\left(1+f_{A P}^{-1} \widehat{f}_{0}\right)\right)$ \\
\hline$H^{\infty}$ & $\underline{\lim } C_{b}\left(\mathbb{A}_{r}\right)$ & & \\
& $C\left(\beta \mathbb{A}_{0} \backslash \mathbb{A}_{0}\right)$ & $\mathbb{Z}$ & {$\left[\left(f_{r}\right)_{r}\right] \mapsto \lim _{r \rightarrow 1} w\left(f_{r}\right)$} \\
\hline
\end{tabular}

TABle 1 . Choices of $S, G, \iota$ corresponding to specific instances of $R$. 
7.1. $\left.R=R H^{\infty}, A(\mathbb{D}), W^{+}(\mathbb{D}), \widehat{L^{1}[0, \infty}\right)+\mathbb{C}, \cdots$. Let

$$
\begin{aligned}
\mathbb{C}_{>0} & :=\{s \in \mathbb{C}: \operatorname{Re}(s)>0\}, \\
\mathbb{D} & :=\{z \in \mathbb{C}:|z|<1\}, \\
\mathbb{T} & :=\{z \in \mathbb{C}:|z|=1\}, \\
\overline{\mathbb{D}} & :=\mathbb{D} \cup \mathbb{T} .
\end{aligned}
$$

Recall that

$$
\begin{aligned}
R H^{\infty} & :=\left\{f: \mathbb{C}_{>0} \rightarrow \mathbb{C}: f \text { is rational and bounded in } \mathbb{C}_{>0}\right\}, \\
A(\mathbb{D}) & :=\{f: \overline{\mathbb{D}} \rightarrow \mathbb{C}: f \text { is holomorphic in } \mathbb{D} \text { and continuous in } \overline{\mathbb{D}}\}, \\
W^{+}(\mathbb{D}) & :=\left\{f: f(z)=\sum_{n=0}^{\infty} a_{n} z^{n}(z \in \overline{\mathbb{D}}) \text { and } \sum_{n=0}^{\infty}\left|a_{n}\right|<+\infty\right\}, \\
\left.L^{\widehat{1}[0, \infty}\right)+\mathbb{C} & :=\left\{f: f(s)=\widehat{f_{\mathrm{a}}}(s)+f_{0}\left(s \in \mathbb{C}_{>0}\right), \text { where } f_{\mathrm{a}} \in L^{1}[0, \infty), f_{0} \in \mathbb{C}\right\} .
\end{aligned}
$$

In the above, $\widehat{f}_{\mathrm{a}}$ denotes the Laplace transform of $f_{\mathrm{a}} \in L^{1}[0, \infty)$. We remark that the set of bounded and holomorphic functions defined in the open right half plane, and which possess a continuous extension to $i \mathbb{R} \cup\left\{\infty_{\mathbb{C}}\right\}$, can be transplanted to functions on the closed unit disk using the conformal map given by

$$
\varphi(z)=\frac{1+z}{1-z} \quad(z \in \mathbb{D}) .
$$

In this manner we may think of elements of $\widehat{L^{1[0, \infty}}+\mathbb{C}$ as being elements of the disc algebra.

In each of these cases, the values of the function on the boundary of the domain of definition gives rise to a function which can be considered to be an element of the $C^{*}$-algebra

$$
S:=C(\mathbb{T}):=\{f: \mathbb{T} \rightarrow \mathbb{C}: f \text { is continuous on } \mathbb{T}\} .
$$

We take $G=\mathbb{Z}$, and $\iota:$ inv $C(\mathbb{T}) \rightarrow \mathbb{Z}$ to be the winding number $w$ with respect to the origin:

$$
\iota(f):=w(f), \quad f \in \operatorname{inv} C(\mathbb{T}) .
$$

Then it can be checked that (A1)-(A3) hold. Most of the details can be found in [1, except for (I5), but this verification is obvious. Moreover, the $\|\cdot\|_{S, \infty}$-norm in the definition of the $d_{c, r}$-metric is the usual supremum $\|\cdot\|_{\infty}$-norm of functions in $C(\mathbb{T})$.

7.2. $R=\mathcal{A}_{+}$. Recall that

$$
\mathcal{A}^{+}=\left\{\begin{array}{l|l}
s\left(\in \mathbb{C}_{>0}\right) \mapsto \widehat{f}_{\mathrm{a}}(s)+\sum_{k=0}^{\infty} a_{k} e^{-s t_{k}} & \begin{array}{l}
f_{\mathrm{a}} \in L^{1}[0, \infty),\left(a_{k}\right)_{k \geq 0} \in \ell^{1}, \\
0=t_{0}<t_{1}, t_{2}, t_{3}, \ldots
\end{array}
\end{array}\right\}
$$

Let

$$
\begin{aligned}
C_{0} & :=\left\{f: \mathbb{R} \rightarrow \mathbb{C}: f \text { is continuous on } \mathbb{R} \text { and } \lim _{x \rightarrow \pm \infty} f(x)=0\right\} \\
A P & :=\text { closed span in } L^{\infty}(\mathbb{R}) \text { of }\left\{(\mathbb{R} \ni) x \mapsto e^{i \lambda x}: \lambda \in \mathbb{R}\right\} .
\end{aligned}
$$


$C_{0}+A P$, endowed with pointwise operations, with the supremum norm, and with involution given by pointwise complex conjugation, is a sub- $C^{*}$-algebra of $L^{\infty}(\mathbb{R})$; 4 . We will take $S:=C_{0}+A P$. Moreover, we take $G=\mathbb{R} \times \mathbb{Z}$, and define $\iota: \operatorname{inv}\left(C_{0}+A P\right) \rightarrow \mathbb{R} \times \mathbb{Z}$ by

$$
\iota(f)=\left(w_{\mathrm{av}}\left(f_{A P}\right), w\left(1+f_{A P}^{-1} f_{0}\right)\right), \quad f=f_{0}+f_{A P} \in \operatorname{inv}\left(C_{0}+A P\right), f_{0} \in C_{0}, f_{A P} \in A P .
$$

In the above, $w_{\mathrm{av}}: \operatorname{inv} A P \rightarrow \mathbb{R}$ denotes the average winding number, defined by

$$
w_{\mathrm{av}}\left(f_{A P}\right):=\lim _{x \rightarrow+\infty} \frac{\arg (f(x))-\arg (f(-x))}{2 x}, \quad f_{A P} \in \operatorname{inv} A P,
$$

see [6. Theorem 1, p. 167]. Again, it can be checked that (A1)-(A3) hold; see [1]. Since $C_{0}+A P$ is a sub- $C^{*}$-algebra of $L^{\infty}(\mathbb{R})$, the $\|\cdot\|_{S, \infty}$-norm in the definition of the $d_{c, r}$-metric is the usual $\|\cdot\|_{\infty}$-norm of functions in $L^{\infty}(\mathbb{R})$.

7.3. $R=H^{\infty}$. The Hardy algebra $H^{\infty}$ consists of all bounded and holomorphic functions defined on the open unit disk $\mathbb{D}:=\{z \in \mathbb{C}:|z|<1\}$, with pointwise operations and the usual supremum norm. We recall the construction of $S$ from [5]. For given $r \in(0,1)$, let

$$
\mathbb{A}_{r}:=\{z \in \mathbb{C}: r<|z|<1\}
$$

denote the open annulus. In particular,

$$
\mathbb{A}_{0}:=\{z \in \mathbb{C}: 0<|z|<1\}
$$

is the punctured disc. Let $C_{b}\left(\mathbb{A}_{r}\right)$ be the $C^{*}$-algebra of all bounded and continuous functions $f: \mathbb{A}_{r} \rightarrow \mathbb{C}$, equipped with pointwise operations and the supremum norm. Moreover, for $0<r \leq R<1$ we define the map $\pi_{r}^{R}: C_{b}\left(\mathbb{A}_{r}\right) \rightarrow C_{b}\left(\mathbb{A}_{R}\right)$ by restriction:

$$
\pi_{r}^{R}(f)=\left.f\right|_{\mathbb{A}_{R}}, \quad f \in C_{b}\left(\mathbb{A}_{r}\right) .
$$

Consider the family $\left(C_{b}\left(\mathbb{A}_{r}\right), \pi_{r}^{R}\right)$ for $0<r \leq R<1$. We note that

(i) $\pi_{r}^{r}$ is the identity map on $C_{b}\left(\mathbb{A}_{r}\right)$, and

(ii) $\pi_{r}^{R} \circ \pi_{\rho}^{r}=\pi_{\rho}^{R}$ for all $0<\rho \leq r \leq R<1$.

Now consider the $*$-algebra

$$
\prod_{r \in(0,1)} C_{b}\left(\mathbb{A}_{r}\right)
$$

and denote by $\mathcal{A}$ its $*$-subalgebra consisting of all elements $f=\left(f_{r}\right)=\left(f_{r}\right)_{r \in(0,1)}$ such that there is an index $r_{0}$ with $\pi_{r}^{R}\left(f_{r}\right)=f_{R}$ for all $0<r_{0} \leq r \leq R<1$. Since every $\pi_{r}^{R}$ is norm decreasing, the net $\left(\left\|f_{r}\right\|_{\infty}\right)$ is convergent and we define

$$
\|f\|:=\lim _{r \rightarrow 1}\left\|f_{r}\right\|_{\infty} .
$$

Clearly this defines a seminorm on $\mathcal{A}$ that satisfies the $C^{*}$-norm identity, that is,

$$
\left\|f^{*} f\right\|=\|f\|^{2}
$$

where $*^{*}$ is the involution, that is, complex conjugation, see (7.2) below. Now, if $N$ is the kernel of $\|\cdot\|$, then the quotient $\mathcal{A} / N$ is a $C^{*}$-algebra (and we denote the norm again by $\|\cdot\|$ ). This algebra is the direct/inductive limit of $\left(C_{b}\left(\mathbb{A}_{r}\right), \pi_{r}^{R}\right)$ and we denote it by

$$
\lim _{\longrightarrow} C_{b}\left(\mathbb{A}_{r}\right) \text {. }
$$


To every element $f \in C_{b}\left(\mathbb{A}_{r_{0}}\right)$, we associate a sequence $f_{1}=\left(f_{r}\right)$ in $\mathcal{A}$, where

$$
f_{r}= \begin{cases}0 & \text { if } 0<r<r_{0}, \\ \pi_{r_{0}}^{r}(f) & \text { if } r_{0} \leq r<1 .\end{cases}
$$

We also define a map $\pi_{r}: C_{b}\left(\mathbb{A}_{r}\right) \rightarrow \underline{\lim } C_{b}\left(\mathbb{A}_{r}\right)$ by

$$
\pi_{r}(f):=\left[f_{1}\right], \quad f \in C_{b}\left(\mathbb{A}_{r}\right),
$$

where $\left[f_{1}\right]$ denotes the equivalence class in $\underline{\lim } C_{b}\left(\mathbb{A}_{r}\right)$ which contains $f_{1}$. We will use the fact that the maps $\pi_{r}$ are in fact $*$-homomorphisms. We note that these maps are compatible with the connecting maps $\pi_{r}^{R}$ in the sense that every diagram shown below is commutative.

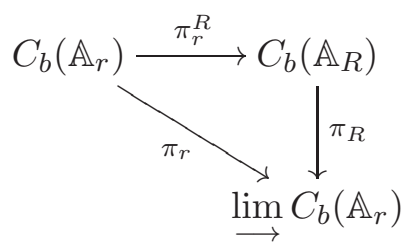

Then $\lim _{\longrightarrow} C_{b}\left(\mathbb{A}_{r}\right)$ is a $C^{*}$-algebra, see [3, Section 2.6]. The multiplicative identity arises from the constant function $f \equiv 1$ in $C_{b}\left(\mathbb{A}_{0}\right)$, that is, $\pi_{0}(f)$. Moreover, we can define an involution in $C_{b}\left(\mathbb{A}_{r}\right)$ by setting

$$
\left(f^{*}\right)(z):=\overline{f(z)}, \quad z \in \mathbb{A}_{r},
$$

and this implicitly defines an involution of elements in $\underset{\lim }{\longrightarrow} C_{b}\left(\mathbb{A}_{r}\right)$. There is a natural embedding of $H^{\infty}$ into $\underset{\lim }{\longrightarrow} C_{b}\left(\mathbb{A}_{r}\right)$, namely

$$
f \mapsto \pi_{0}(f): H^{\infty} \longrightarrow \lim _{\longrightarrow} C_{b}\left(\mathbb{A}_{r}\right) .
$$

We will take $G=\mathbb{Z}$. For $f \in \operatorname{inv}\left(C_{b}\left(\mathbb{A}_{\rho}\right)\right)$ and for $0<\rho<r<1$ we define the map $f^{r}: \mathbb{T} \rightarrow \mathbb{C}$ by $f^{r}(\zeta)=f(r \zeta), \zeta \in \mathbb{T}$. If $f \in \operatorname{inv}\left(C_{b}\left(\mathbb{A}_{\rho}\right)\right)$, then $f^{r} \in \operatorname{inv}(C(\mathbb{T}))$, and so $f^{r}$ has a winding number $w\left(f_{r}\right)$. We set $w(f):=w\left(f^{r}\right) \in \mathbb{Z}$ with respect to 0 , and it can be shown that this is well-defined. Now we define the map $\iota: \operatorname{inv}\left(\underline{\lim } C_{b}\left(\mathbb{A}_{r}\right)\right) \rightarrow \mathbb{Z}$. For $\left[\left(f_{r}\right)\right] \in \operatorname{inv}\left(\stackrel{\lim }{\longrightarrow} C_{b}\left(\mathbb{A}_{r}\right)\right)$,

$$
\iota(f):=\lim _{r \rightarrow 1} w\left(f_{r}\right), \text { for } f=\left[\left(f_{r}\right)\right] \in \operatorname{inv}\left(\underline{\lim } C_{b}\left(\mathbb{A}_{r}\right)\right) .
$$

It can be shown that $\iota$ is well-defined and all the properties we demand are satisfied; see [5]. It was also shown there that $\lim _{\longrightarrow} C_{b}\left(\mathbb{A}_{r}\right)$ is isometrically isomorphic to $C\left(\beta \mathbb{A}_{0} \backslash \mathbb{A}_{0}\right)$ (where

$$
\beta \mathbb{A}_{0}:=\text { Stone-Čech compactification of } \mathbb{A}_{0},
$$

which is the maximal ideal space of the Banach algebra $C_{b}\left(\mathbb{A}_{0}\right)$ of all complex-valued bounded continuous functions on $\mathbb{A}_{0}$ ), and moreover $\lim _{\longrightarrow} C_{b}\left(\mathbb{A}_{r}\right)$ is a sub- $C^{*}$-algebra of $L^{\infty}(\mathbb{T})$. From here we see that the $\|\cdot\|_{S, \infty}$-norm in the definition of the $d_{c, r}$-metric is the usual $\|\cdot\|_{\infty}$-norm of functions in $L^{\infty}(\mathbb{R})$.

\section{An EXAMPle}

As an illustration, we consider [8, Example 4.3], namely

$$
\mathbf{p}_{1}=\frac{1}{s-e^{-s}} \text {. }
$$


It was shown in 8 that an algebraic spectral factorization leading to normalized coprime factors is not possible in this example. So calculating the $\nu$-metric between this plant and a perturbed one, say

$$
\mathbf{p}_{a}:=\frac{1}{s-a e^{-s}},
$$

is problematic. Nevertheless, there exists a coprime factorization of

$$
\mathbf{p}_{a}=\frac{\mathbf{n}_{a}}{\mathbf{d}_{a}}
$$

over $H^{\infty}$ of the half-plane $\mathbb{C}_{>0}$, where

$$
\mathbf{n}_{a}:=\frac{1}{1+s}, \quad \mathbf{d}_{a}:=\frac{s-a e^{-s}}{1+s} .
$$

That this is a coprime factorization over $H^{\infty}$ follows from the fact that

$$
\begin{aligned}
& \mathbf{x}:=1+e^{-s}, \\
& \mathbf{y}:=1
\end{aligned}
$$

solve the Bezout equation $\mathbf{n} \mathbf{x}+\mathbf{d y}=1$ :

$$
\mathbf{n} \mathbf{x}+\mathbf{d y}=\frac{1}{1+s} \cdot\left(1+e^{-s}\right)+\frac{s-e^{-s}}{1+s} \cdot 1=1
$$

We will see that using this coprime factorization, we can compute $d_{c, r}\left(\mathbf{p}_{a}, \mathbf{p}_{1}\right)$ fairly easily. We remark also that $\mathbf{p}_{a}$ is the transfer function associated with the retarded delay differential equation

$$
\left.\begin{array}{rl}
x^{\prime}(t) & =a \cdot x(t-1)+u(t), \\
y(t) & =x(t)
\end{array}\right\}, \quad t \geq 0 .
$$

For $s \in \mathbb{C}_{>0}$, and with $a=: 1+\delta$, we have

$$
\overline{\mathbf{n}_{1}(s)} \cdot \mathbf{d}_{a}(s)+\overline{\mathbf{d}_{1}(s)} \cdot \mathbf{n}_{a}(s)=\underbrace{\frac{1}{|1+s|^{2}}+\frac{\left|s-e^{-s}\right|^{2}}{|1+s|^{2}}}_{=: f_{1}(s)}-\delta \cdot \underbrace{\frac{e^{-s}\left(\bar{s}-e^{-\bar{s}}\right)}{|1+s|^{2}}}_{=: f_{2}(s)} .
$$

It is easy to see that since $\lim _{\substack{s \in \mathbb{C}>0 \\ s \rightarrow \infty}} f_{1}(s)=1$, and for all $s \in \mathbb{C}_{>0}, f_{1}(s) \geq \frac{1}{|1+s|^{2}}>0$, we have

$$
m:=\inf _{s \in \mathbb{C}_{>0}} f_{1}(s)>0 .
$$

Also, $\lim _{\substack{s \in \mathbb{C}>0 \\ s \rightarrow \infty}} f_{2}(s)=0$, and so

$$
M:=\sup _{s \in \mathbb{C}_{>0}}\left|f_{2}(s)\right|<+\infty .
$$

Consequently for all $\delta=a-1$ small enough, for example, $|\delta|=|a-1|<\frac{m}{2(M+1)}$, we have

$$
\operatorname{Re}\left(\overline{\mathbf{n}_{1}(s)} \cdot \mathbf{d}_{a}(s)+\overline{\mathbf{d}_{1}(s)} \cdot \mathbf{n}_{a}(s)\right)>m-|\epsilon| M>m-\frac{m}{2(M+1)} \cdot M>m-\frac{m}{2}=\frac{m}{2}>0 .
$$


So the condition that $\mathbf{n}_{1}^{*} \mathbf{d}_{a}+\mathbf{d}_{1}^{*} \mathbf{n}_{a} \in \operatorname{inv} S$ and $\iota\left(\mathbf{n}_{1}^{*} \mathbf{d}_{a}+\mathbf{d}_{1}^{*} \mathbf{n}_{a}\right)=0$ will be satisfied for all such values of the parameter $a$. Hence

$$
\begin{aligned}
d_{c, r}\left(\mathbf{p}_{1}, \mathbf{p}_{a}\right) & =\sup _{y \in \mathbb{R}} \frac{\left|\mathbf{n}_{1}(i y) \mathbf{d}_{a}(i y)-\mathbf{d}_{1}(i y) \mathbf{n}_{a}(i y)\right|}{\sqrt{\mid \mathbf{n}_{1}\left(\left.i y\right|^{2}+\left|\mathbf{d}_{1}(i y)\right|^{2}\right.} \sqrt{\left|\mathbf{n}_{a}(i y)\right|^{2}+\left|\mathbf{d}_{a}(i y)\right|^{2}}} \\
& =\sup _{y \in \mathbb{R}} \frac{\frac{|a-1|}{1+y^{2}}}{\sqrt{\frac{1+y^{2}+1+2 y \sin y}{1+y^{2}}} \sqrt{\frac{1+y^{2}+a^{2}+2 a y \sin y}{1+y^{2}}}} \\
& =\frac{|a-1|}{\sqrt{2\left(1+a^{2}\right)}},
\end{aligned}
$$

for all $a \in \mathbb{R}$ satisfying

where $m, M$ are given by

$$
|a-1|<\frac{m}{2(M+1)}
$$

$$
\begin{aligned}
m & :=\inf _{s \in \mathbb{C}>0}\left(\frac{1}{|1+s|^{2}}+\frac{\left|s-e^{-s}\right|^{2}}{|1+s|^{2}}\right), \\
M & :=\sup _{s \in \mathbb{C}>0} \frac{\left|e^{-s}\left(\bar{s}-e^{-\bar{s}}\right)\right|}{|1+s|^{2}} .
\end{aligned}
$$

It is clear that $M \geq 0$ and

$$
m \leq 2=\left.\left(\frac{1}{|1+s|^{2}}+\frac{\left|s-e^{-s}\right|^{2}}{|1+s|^{2}}\right)\right|_{s=0},
$$

so that

$$
d_{c, r}\left(\mathbf{p}_{1}, \mathbf{p}_{a}\right)=\frac{|a-1|}{\sqrt{2\left(1+a^{2}\right)}}
$$

for all $a \in \mathbb{R}$ satisfying $|a-1|<1$.

We remark that a stabilizing controller for the nominal plant $\mathbf{p}_{1}$ is trivially found to be

$$
\mathbf{c}=-\frac{\mathbf{x}}{\mathbf{y}}
$$

where $\mathbf{x}:=1+e^{-s}$ and $\mathbf{y}:=1$ (which solve the Bezout equation $\mathbf{n x}+\mathbf{d y}=1$ ). The stability margin of the pair $\left(\mathbf{p}_{1}, \mathbf{c}\right)$ is given by

$$
\begin{aligned}
\mu_{\mathbf{p}_{1}, \mathbf{c}}^{-1} & =\left\|H\left(\mathbf{p}_{1}, \mathbf{c}\right)\right\|_{\infty}=\left\|\left[\begin{array}{cc}
\frac{1+e^{-s}}{1+s} & \frac{1}{1+s} \\
\frac{\left(1+e^{-s}\right)\left(s-e^{-s}\right)}{1+s} & \frac{s-e^{-s}}{1+s}
\end{array}\right]\right\|_{\infty} \\
& =\left\|\left[\begin{array}{c}
\frac{1}{1+s} \\
\frac{s-e^{-s}}{1+s}
\end{array}\right]\left[1+e^{-s} 1\right]\right\|_{\infty} .
\end{aligned}
$$

It follows that

$$
\mu_{\mathbf{p}_{1}, \mathbf{c}}^{-1}=\sup _{s \in i \mathbb{R}} \sqrt{\left(\left|\frac{1}{1+s}\right|^{2}+\left|\frac{s-e^{-s}}{1+s}\right|^{2}\right)\left(\left|1+e^{-s}\right|^{2}+1^{2}\right)} .
$$


A crude upper bound for $\mu_{\mathbf{p}_{1}, \mathbf{c}}^{-1}$ can be shown to be 5 (a Maple plot reveals the value being around 3.224):

$$
\begin{aligned}
\mu_{\mathbf{p}_{1}, \mathbf{c}}^{-1} & =\sup _{y \in \mathbb{R}} \sqrt{\frac{2+2 y \sin y+y^{2}}{1+y^{2}}(3+2 \cos y)} \leq \sup _{y \in \mathbb{R}} \sqrt{\frac{2+2 y^{2}+y^{2}}{1+y^{2}}(3+2 \cdot 1)} \\
& \leq \sup _{y \in \mathbb{R}} \sqrt{2 \cdot \frac{1}{1+y^{2}}+3 \cdot \frac{y^{2}}{1+y^{2}}} \sqrt{5} \leq \sqrt{2 \cdot 1+3 \cdot 1} \sqrt{5}=5 .
\end{aligned}
$$

Thus $\mu_{\mathbf{p}_{1}, \mathbf{c}} \geq 1 / 5$. From Theorem 4.5, it follows that for all $a \in \mathbb{R}$ such that $|a-1|<1$, and with

$$
d_{c, r}\left(\mathbf{p}, \mathbf{p}_{1}\right)=\frac{|a-1|}{\sqrt{2\left(1+a^{2}\right)}}<\frac{1}{5}
$$

$\mathbf{p}$ is stabilized by $\mathbf{c}$, guaranteeing a stability margin of

$$
\frac{1}{5}-\frac{|a-1|}{\sqrt{2\left(1+a^{2}\right)}}>0
$$

We remark that the inequality

$$
\frac{|a-1|}{\sqrt{2\left(1+a^{2}\right)}}<\frac{1}{5}
$$

is satisfied by all $a \in \mathbb{R}$ in the interval $(2 / 3,3 / 2)$, and then also $|a-1|<1$. So all plants

$$
\mathbf{p}=\frac{1}{s-a e^{-s}}
$$

with $a \in(2 / 3,3 / 2)$ are stabilized by the controller $\mathbf{c}=-\left(1+e^{-s}\right)$ which stabilizes the nominal plant

$$
\mathbf{p}_{1}=\frac{1}{s-e^{-s}}
$$

Acknowledgements: The author thanks the two referees for their comments which greatly improved the presentation of the article.

\section{REFERENCES}

[1] J.A. Ball and A.J. Sasane. Extension of the $\nu$-metric. Complex Analysis and Operator Theory, 6:65-89, 2012.

[2] J.A. Ball and A.J. Sasane. Extension of the $\nu$-metric: the $H^{\infty}$ case. In Spectral theory, mathematical system theory, evolution equations, differential and difference equations, 121130, Oper. Theory Adv. Appl., 221, Birkhäuser/Springer Basel AG, Basel, 2012.

[3] Lectures on operator theory. Edited by R. Bhat, G.A. Elliott, P.A. Fillmore. Fields Institute Monographs, 13, American Mathematical Society, Providence, RI, 1999.

[4] R.G. Douglas. On the $C^{*}$-algebra of a one-parameter semigroup of isometries. Acta Mathematica, 128:143151, no. 3-4, 1972.

[5] M. Frentz and A.J. Sasane. Reformulation of the extension of the $\nu$-metric for $H^{\infty}$. Journal of Mathematical Analysis and Applications , 401:659-671, no. 2, 2013.

[6] B. Jessen and H. Tornehave. Mean motions and zeros of almost periodic functions. Acta Mathematica, 77:137-279, 1945.

[7] K.M. Mikkola. Real solutions to control, approximation, and factorization problems. SIAM J. Control Optim., 50:1071-1086, no. 3, 2012.

[8] J.R. Partington and G.K. Sankaran. Algebraic construction of normalized coprime factors for delay systems. Mathematics of Control, Signals, and Systems, 15:1-12, 2002. 
[9] A. Quadrat. The fractional representation approach to synthesis problems: an algebraic analysis viewpoint. Part I: (Weakly) doubly coprime factorizations. SIAM Journal on Control and Optimization, 42:266-299, 2004.

[10] A. Quadrat. The fractional representation approach to synthesis problems: an algebraic analysis viewpoint. II. Internal stabilization. SIAM J. Control Optim., 42:300-320, no. 1, 2003.

[11] W. Rudin. Functional analysis. Second Edition. Tata McGraw-Hill, New Delhi, 2006.

[12] A.J. Sasane. Extension of the $\nu$-metric for stabilizable plants over $H^{\infty}$. Math. Control Relat. Fields, 2:29-44, no. 1, 2012.

[13] A.J.Sasane. A Generalized Chordal Metric in Control Theory Making Strong Stabilizability a Robust Property. Complex Analysis and Operator Theory, to appear.

[14] S. Treil. A counterexample on continuous coprime factors. IEEE Transactions on Automatic Control, no. 6, 39:1262-1263, 1994.

[15] M. Vidyasagar. Control system synthesis. A factorization approach. MIT Press, Cambridge, MA, 1985.

[16] G. Vinnicombe. Frequency domain uncertainty and the graph topology. IEEE Transactions on Automatic Control, no. 9, 38:1371-1383, 1993.

Department of Mathematics, Faculty of Science, Lund University, Sweden.

E-mail address: amol.sasane@math.lu.se 